levels of received support remained significantly associated with a lower likelihood of perinatal depression in migrants (adjusted odds ratio $0.82 ; 95 \%$ CI $0.68-0.99$ ). In the final model for refugees, all three social support measures were dropped from the model. Among both migrants and refugees, a previous history of depression and experiences of trauma were strongly associated with perinatal depression after controlling for all other variables.

Conclusion Our findings highlight the importance of received social support to perinatal depression in migrant women on the Thailand-Myanmar border. Experience of trauma and prior depression also strongly predicted perinatal depression. The perinatal period offers a valuable window of opportunity to ask women about their mental health. Our results suggest women should also be asked about support networks available, trauma and past episodes of depression. Future research should focus on testing community-level and public policy interventions to nurture support networks for migrant and refugee women in their resettlement destinations.

\section{OP57 VALIDATED SCREENING TOOLS FOR COMMON MENTAL DISORDERS IN PERINATAL WOMEN IN INDIA: A SYSTEMATIC REVIEW AND META-ANALYSIS}

${ }^{1}$ Gracia Fellmeth* ${ }^{1}$ Siân Harrison, ${ }^{1}$ Charles Opondo, ${ }^{1}$ Manisha Nair, ${ }^{1}$ Jennifer J Kunceuk, 1,2Fiona Alderdice. 'National Perinatal Epidemiology Unit, Nuffield Department of Population Health, University of Oxford, Oxford, UK; ${ }^{2}$ School of Nursing and Midwifery, Queens University Belfast, UK

\subsection{6/jech-2021-SSMabstracts.57}

Background Common mental disorders (CMD) in the perinatal period are associated with significant adverse outcomes for women and their families. Prevalence of perinatal CMD is significant in low- and middle-income countries such as India, where women living in socio-economic deprivation, those experiencing intimate partner violence and those with low societal status are at high risk of perinatal mental disorders. Early detection and treatment of perinatal CMD can minimise adverse effects and improve outcomes. However, tools must be validated against a gold-standard locally to assess their psychometric validity and ensure they are culturally appropriate. We aimed to systematically review and synthesise the current evidence on screening tools for perinatal CMD that have been validated in India.

Methods We searched MEDLINE, Embase, PsycINFO, Global Health, Cochrane Library, Web of Science and Google Scholar without language or date restrictions using search terms for CMD, perinatal status, screening and India. Studies assessing the validity of a screening tool for CMD against a gold standard in women who were pregnant or up to 12 months postpartum in India was included. Two reviewers independently screened titles, abstracts and full-texts and extracted data. Study quality was assessed using the QUADAS-2 tool. We used bivariate and hierarchical summary receiver operating characteristic models to calculate pooled summary estimates of sensitivity and specificity.

Results We identified 8306 records of which 2838 were duplicates, 5390 were excluded by title and abstract and 76 were excluded by full-text. Five additional records were identified through grey literature searches. Seven studies were included in the review of which six (1003 participants) were included in meta-analysis. All included studies assessed the validity of the Edinburgh Postnatal Depression Scale (EPDS) in identifying perinatal depression. No studies tested the validity of screening tools for perinatal anxiety. At a threshold of $\geq 13$ the EPDS had a pooled sensitivity of $88.9 \% \quad(95 \%$ CI 77.4-94.9) and pooled specificity of 93.4 (95\% CI $81.5-$ 97.8). Two studies had a low risk of bias across all domains; all others had unclear or high risk of bias across at least one domain.

Conclusion The EPDS appears to be psychometrically valid for identifying perinatal depression in India. There is a scarcity of evidence around the validity of other screening tools for perinatal CMD in India, with no studies of screening tools for perinatal anxiety identified. Evidence reviews should include rigorous searches of the grey literature to avoid missing studies published in non-indexed journals. Further research is required to inform decisions around screening tools for the identification of women with perinatal CMD in India.

\section{OP58 PREVALENCE OF PERINATAL ANXIETY IN LOW- AND MIDDLE-INCOME COUNTRIES: A SYSTEMATIC REVIEW AND META-ANALYSIS}

${ }^{1}$ Maria Nielsen-Scott ${ }^{*},{ }^{2}$ Gracia Fellmeth, ${ }^{2}$ Charles Opondo, ${ }^{2,3}$ Fiona Alderdice. ${ }^{1}$ Medical School, University of Oxford, Oxford, UK; ${ }^{2}$ National Perinatal Epidemiology Unit, University of Oxford, Oxford, UK; ${ }^{3}$ School of Nursing and Midwifery, Queens University Belfast, Belfast, UK

\subsection{6/jech-2021-SSMabstracts.58}

Background Perinatal anxiety is associated with adverse outcomes for women and their infants. Women in low- and middle-income countries (LMIC) may be at higher risk of perinatal anxiety disorders.

Aims We aim to systematically review the evidence on prevalence of perinatal anxiety and calculate pooled prevalence estimates of antenatal and postnatal anxiety among women living in LMIC.

Method We searched MEDLINE, Embase, PscyhINFO, Global Health and Web of Science to identify studies assessing prevalence of perinatal anxiety in LMIC. Studies published since January 2016 were included. Screening and data extraction was conducted independently by two reviewers. Pooled prevalence estimates were calculated using random-effect meta-analyses and sources of heterogeneity explored through subgroup analyses and meta-regression.

Results We screened 9494 titles and abstracts and reviewed 710 full-texts. We included 56 publications (54 studies) in the systematic review and 54 in meta-analysis. The pooled prevalence of self-reported anxiety symptoms was 29.2\% (95\% CI $24.5-34.2 ; \mathrm{I} 298.7 \% ; 36$ studies; $\mathrm{n}=28,755)$ antenatally and 24.4\% (95\% CI 16.2-33.7; I2 98.5\%; 15 studies; $\mathrm{n}=6370$ ) postnatally. The prevalence of clinically-diagnosed anxiety disorder was $8.1 \% \quad(95 \%$ CI $4.4-12.8 ; \quad$ I2 $88.1 \% \quad 5$ studies; $\mathrm{n}=1659)$ antenatally and $16.0 \%(95 \%$ CI $13.5-18.9 ; \mathrm{n}=113)$ postnatally.

Conclusion Perinatal anxiety represents a significant burden in LMIC, with one in four women experiencing symptoms during pregnancy or postpartum. Research remains lacking in a significant proportion of LMIC, particularly in the lowest income countries. Further research should guide application of screening tools in clinical settings to identify women with anxiety disorders in order to provide appropriate treatment. 